\title{
Non-simultaneous blow-up for a parabolic system with nonlinear boundary flux which obey different laws
}

\section{Si $\mathrm{Xu}^{1 *}$ and Jinfa Zeng ${ }^{2}$}

"Correspondence:

xusi_math@hotmail.com 'Department of Mathematics, Jiangxi Vocational College of Finance and Economics, Jiujiang, Jiangxi 332000, P.R. China Full list of author information is available at the end of the article

\begin{abstract}
In this paper, we consider a system of two heat equations with nonlinear boundary flux which obey different laws, one is exponential nonlinearity and another is power nonlinearity. Under certain hypotheses on the initial data, we get the sufficient and necessary conditions, on which there exist initial data such that non-simultaneous blow-up occurs. Moreover, we get some conditions on which simultaneous blow-up must occur. Furthermore, we also get a result on the coexistence of both simultaneous and non-simultaneous blow-ups.
\end{abstract}

MSC: 35B33; 35K65; 35K55

Keywords: simultaneous blow-up; non-simultaneous blow-up; parabolic system; nonlinear boundary flux

\section{Introduction and main results}

In this paper, we study the following system of two heat equations coupled by nonlinear boundary conditions,

$$
\left\{\begin{array}{l}
u_{t}=\Delta u, \quad v_{t}=\Delta v, \quad(x, t) \in \Omega \times(0, T), \\
\frac{\partial u}{\partial \eta}=e^{p v} u^{\alpha}, \quad \frac{\partial v}{\partial \eta}=u^{q} e^{\beta v}, \quad(x, t) \in \partial \Omega \times(0, T), \\
u(x, 0)=u_{0}(x), \quad v(x, 0)=v_{0}(x), \quad x \in \Omega,
\end{array}\right.
$$

where $\Omega=B_{R}=\{|x|<R\} \subset \mathbb{R}^{N}$, parameters $\alpha, q \geq 1, p, \beta \geq 0$. Assume the non-zero, nonnegative initial data $u_{0}, v_{0}$ are radially symmetric non-increasing continuous functions, vanishing on $\partial \Omega$, as well as satisfy the compatibility conditions,

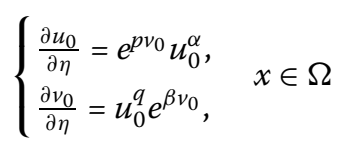

and $\Delta u_{0}, \Delta v_{0} \geq 0$, for $x \in \Omega$.

The system (1.1) can be used to describe heat propagation of a two-component combustible mixture in a bounded region. In this case, $u$ and $v$ represent the temperatures of the interacting components, thermal conductivity is supposed constant and equal for both substances, and a volume energy release given by powers of $u$ and $v$ is assumed; see

\section{Springer}

(C) 2012 Xu and Zeng; licensee Springer. This is an Open Access article distributed under the terms of the Creative Commons Attribution License (http://creativecommons.org/licenses/by/2.0), which permits unrestricted use, distribution, and reproduction in any medium, provided the original work is properly cited. 
$[1,6]$. The nonlinear Neumann boundary conditions can be physically interpreted as the cross-boundary fluxes, which obey different laws; some may obey power laws $[4,7,10,14]$, some may follow exponential laws [18]. It is interesting when the two types of boundary fluxes meet. In system (1.1), the coupled boundary flux obey a mixed type of power terms and exponential terms.

As we shall see, under certain conditions the solutions of this problem can become unbounded in a finite time. This phenomenon is known as blow-up, and has been observed for several scalar equation since the pioneering work of Fujita. Blow-up may also happen for systems, X. F. Song considered the blow-up conditions and blow-up rates of system (1.1), when $p, q>0,0 \leq \alpha<1$ and $0 \leq \beta<p$, in [16].

However, it can only show

$$
\lim _{t \rightarrow T} \sup \left\{\|u(\cdot, t)\|_{\infty}+\|v(\cdot, t)\|_{\infty}\right\}=\infty
$$

whether the blow-up is simultaneous or non-simultaneous is not known yet.

Recently, the simultaneous and non-simultaneous blow-up problems of parabolic systems have been widely considered by many authors $[2,3,8,9,11-13,15,19,20]$. For example, B. C. Liu and F. J. Li [8] considered the nonlinear parabolic system

$$
\left\{\begin{array}{l}
u_{t}=\Delta u+u^{m} e^{p v}, \quad v_{t}=\Delta v+u^{q} e^{n v}, \quad(x, t) \in \Omega \times(0, T), \\
u(x, t)=v(x, t)=0, \quad(x, t) \in \partial \Omega \times(0, T), \\
u(x, 0)=u_{0}(x), \quad v(x, 0)=v_{0}(x), \quad x \in \Omega .
\end{array}\right.
$$

They got a complete and optimal classification on non-simultaneous and simultaneous blow-ups by four sufficient and necessary conditions.

Motivated by the above works, we will focus on the simultaneous and non-simultaneous blow-up problems to system (1.1), and get our main results as follows.

Theorem 1.1 There exist initial data such that the solutions of (1.1) blow up, if

$$
\alpha>1, \quad \text { or } \beta>0, \quad \text { or } \quad p q>\beta(\alpha-1) \text {. }
$$

In the sequel, we assume the blow-up indeed occurs. Then we get the conditions, under which simultaneous or non-simultaneous blow-up occurs.

Theorem 1.2 There exist initial data such that non-simultaneous blow-up occurs if and only if

$$
\alpha>q+1, \quad \text { or } \quad \beta>p
$$

Corollary 1.1 Any blow-up is simultaneous if and only if

$$
\left\{\begin{array}{l}
\alpha \leq q+1 \\
\beta \leq p
\end{array}\right.
$$




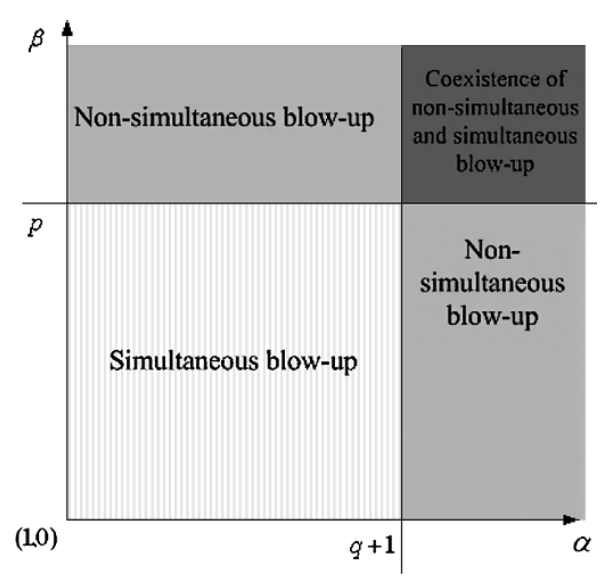

Figure 1 Non-simultaneous and simultaneous blow-ups.

\section{Theorem 1.3 If}

$$
\left\{\begin{array}{l}
\alpha>q+1, \\
\beta>p
\end{array}\right.
$$

both non-simultaneous and simultaneous blow-ups may occur.

In order to show the conditions more clearly, we graph Figure 1 with the region of nonsimultaneous and simultaneous blow-ups occur in the parameter space.

The rest of this paper is organized as follows: In next section, we consider the blowup conditions of system (1.1), give the proof of Theorem 1.1. In Section 3, we will study the sufficient and necessary conditions of non-simultaneous blow-up, in order to prove Theorem 1.2. In Section 4, we consider the coexistence of both simultaneous and nonsimultaneous blow-ups; Theorem 1.3 is proved.

\section{Blow-up}

In this section, we prove the blow-up criterion of system (1.1). First, we check the monotonicity of the solution.

Lemma 2.1 Let $(u, v)$ be a solution of system $(1.1)$, then $u_{t}, v_{t} \geq 0$, for all $(x, t) \in B_{R} \times(0, T)$.

Proof Set

$$
M=u_{t}, \quad N=v_{t}, \quad(x, t) \in B_{R} \times(0, T) .
$$

From the hypothesis of initial data, we can get

$$
\left\{\begin{array}{l}
M_{t}=\Delta M, \quad N_{t}=\Delta N, \quad(x, t) \in B_{R} \times(0, T), \\
\frac{\partial M}{\partial \eta}=p u^{\alpha} e^{p v} N+\alpha u^{\alpha-1} e^{p v} M, \\
\frac{\partial N}{\partial \eta}=q u^{q-1} e^{\beta v} M+\beta u^{q} e^{\beta v} N, \quad(x, t) \in \partial B_{R} \times(0, T), \\
M(x, 0)=\Delta u_{0} \geq 0, \quad N(x, 0)=\Delta v_{0} \geq 0, \quad x \in B_{R} .
\end{array}\right.
$$

By the comparison principle, $M(x, t), N(x, t) \geq 0$, for $(x, t) \in B_{R} \times(0, T)$. 
Proof of Theorem 1.1 It is easy to check that

$$
\left\{\begin{array}{l}
\frac{\partial u}{\partial \eta}=e^{p v} u^{\alpha} \geq v^{p} u^{\alpha} \\
\frac{\partial v}{\partial \eta}=u^{q} e^{\beta v} \geq u^{q} \cdot\left(\frac{\beta}{\beta+1}\right)^{\beta+1} \cdot v^{\beta+1} .
\end{array}\right.
$$

Let $(\underline{u}, \underline{v})$ be a solution of the following system:

$$
\left\{\begin{array}{l}
\underline{u}_{t}=\Delta \underline{u}, \quad \underline{v}_{t}=\Delta \underline{v}, \quad(x, t) \in \Omega \times(0, T), \\
\frac{\partial \underline{u}}{\partial \eta}=\underline{u}^{\alpha} \underline{v}^{\beta}, \quad \frac{\partial \underline{v}}{\partial \eta}=\left(\frac{\beta}{\beta+1}\right)^{\beta+1} \underline{u}^{q} \underline{v}^{\beta+1}, \quad(x, t) \in \partial \Omega \times(0, T), \\
\underline{u}(x, 0)=u_{0}(x), \quad \underline{v}(x, 0)=v_{0}(x), \quad x \in \Omega .
\end{array}\right.
$$

By the results of [17], the solutions of (2.1) blow up with large initial data if $\alpha>1$, or $\beta>0$, or $p q>\beta(\alpha-1)$. By the comparison principle, $(\underline{u}, \underline{v})$ is a sub-solution of (1.1), thus the solutions of (1.1) also blow up.

\section{Non-simultaneous blow-up}

In this section, we prove Theorem 1.2 with four lemmas. Firstly, we define the set of initial data with a fixed constant $\varepsilon \in(0,1)$,

$$
\mathbb{V}_{0}=\left\{\left(u_{0}, v_{0}\right) \mid \Delta u_{0}-\varepsilon u_{0}^{\alpha} e^{p v_{0}} \geq 0, \Delta v_{0}-\varepsilon u_{0}^{q} e^{\beta v_{0}} \geq 0, x \in B_{R}\right\}
$$

Lemma 3.1 For any $\left(u_{0}, v_{0}\right) \in \mathbb{V}_{0}$, there must be

$$
\begin{aligned}
& u_{t}(x, t) \geq \varepsilon\left(u^{\alpha} e^{p v}\right)(x, t) \\
& v_{t}(x, t) \geq \varepsilon\left(u^{q} e^{\beta v}\right)(x, t)
\end{aligned} \quad(x, t) \in B_{R} \times[0, T) .
$$

Proof Set

$$
J=u_{t}-\varepsilon u^{\alpha} e^{p v}, \quad K=v_{t}-\varepsilon u^{q} e^{\beta v}, \quad(x, t) \in B_{R} \times[0, T) .
$$

By computations, we can check that

$$
\begin{gathered}
J_{t}-\Delta J=\left(u_{t}-\Delta u\right)_{t}-\varepsilon \alpha u^{\alpha-1} e^{p v}\left(u_{t}-\Delta u\right)-\varepsilon p u^{q} e^{p v}\left(v_{t}-\Delta v\right) \\
+\varepsilon u^{\alpha-2} e^{p v}\left(\alpha(\alpha-1) u_{r}^{2}+2 \alpha p u u_{r} v_{r}+p^{2} u^{2} v_{r}^{2}\right) \geq 0, \\
K_{t}-\Delta K=\left(v_{t}-\Delta v\right)_{t}-\varepsilon q u^{q-1} e^{\beta v}\left(u_{t}-\Delta u\right)-\varepsilon \beta u^{q} e^{\beta v}\left(v_{t}-\Delta v\right) \\
\quad+\varepsilon u^{q-2} e^{\beta v}\left(q(q-1) u_{r}^{2}+2 q \beta u u_{r} v_{r}+\beta^{2} u^{2} v_{r}^{2}\right) \geq 0, \\
(x, t) \in B_{R} \times(0, T) . \\
\frac{\partial J}{\partial \eta}=\left(e^{p v} u^{\alpha}\right)_{t}-\varepsilon \alpha u^{\alpha-1} \frac{\partial u}{\partial \eta} e^{\beta v}-\varepsilon u^{\alpha} e^{p v} p \frac{\partial v}{\partial \eta} \\
=e^{p v} p u^{\alpha}\left(v_{t}-\varepsilon u^{q} e^{\beta v}\right)+e^{p v} \alpha u^{\alpha-1}\left(u_{t}-\varepsilon u^{\alpha} e^{p v}\right) \\
=p u^{\alpha} e^{p v} K+\alpha u^{\alpha-1} e^{p v} J, \\
\frac{\partial K}{\partial \eta}=q u^{q-1} e^{\beta v} J+\beta u^{q} e^{\beta v} K, \quad(x, t) \in \partial B_{R} \times(0, T) .
\end{gathered}
$$




$$
\begin{aligned}
& J(x, 0)=\Delta u_{0}-\varepsilon u_{0}^{\alpha} e^{p v_{0}} \geq 0, \quad x \in B_{R}, \\
& K(x, 0)=\Delta v_{0}-\varepsilon u_{0}^{q} e^{\beta v_{0}} \geq 0, \quad x \in B_{R} .
\end{aligned}
$$

By the comparison principle, $J(x, t), K(x, t) \geq 0$, for $(x, t) \in B_{R} \times[0, T)$.

Lemma 3.2 For any $t \in[0, T)$

$$
\begin{aligned}
& u(0, t) \leq\left[(\alpha-1) \varepsilon e^{p v_{0}(0)}\right]^{-\frac{1}{\alpha-1}}(T-t)^{-\frac{1}{\alpha-1}} \quad(\alpha \neq 1), \\
& v(0, t) \leq \ln \left\{\left[\beta \varepsilon u_{0}^{q}(0)\right]^{-\frac{1}{\beta}}(T-t)^{-\frac{1}{\beta}}\right\} \quad(\beta \neq 0) .
\end{aligned}
$$

Proof First, we prove (3.2). From (3.1), we get

$$
u_{t}(0, t) \geq \varepsilon u^{\alpha}(0, t) e^{p v(0, t)},
$$

then

$$
u_{t}(0, t) \geq \varepsilon u^{\alpha}(0, t) e^{p v_{0}(0, t)}
$$

Integrating (3.4) from $t$ to $T$,

$$
\int_{t}^{T} \frac{u_{t}(0, t) d t}{u^{\alpha}(0, t)} \geq \varepsilon e^{p v_{0}(0)}(T-t),
$$

thus

$$
-u^{-\alpha+1}(0, t) \geq \varepsilon e^{p v_{0}(0)}(T-t)(-\alpha+1),
$$

then

$$
u(0, t) \leq\left[(\alpha-1) \varepsilon e^{p v_{0}(0)}\right]^{-\frac{1}{\alpha-1}}(T-t)^{-\frac{1}{\alpha-1}} .
$$

Similarly, we can also prove (3.3) from (3.1),

$$
v_{t}(0, t) \geq \varepsilon u^{q}(0, t) e^{\beta v(0, t)} \geq \varepsilon u_{0}^{q}(0) e^{\beta v(0, t)} .
$$

Integrating the above inequality from $t$ to $T$, then

$$
\begin{aligned}
& \int_{t}^{T} e^{-\beta v(0, t)} v_{t}(0, t) d t \geq \varepsilon u_{0}^{q}(0)(T-t), \\
& \frac{1}{\beta} e^{-\beta v(0, t)} \geq \varepsilon u_{0}^{q}(0)(T-t), \\
& v(0, t) \leq-\frac{1}{\beta} \ln \left[\beta \varepsilon u_{0}^{q}(0)(T-t)\right] .
\end{aligned}
$$

The following lemma proves the sufficient and necessary condition on the existence of $u$ blowing up alone.

Lemma 3.3 There exist suitable initial data such that $u$ blows up while v remains bounded if and only if $\alpha>q+1$. 
Proof Firstly, we prove the sufficiency.

Let

$$
\Gamma(x, y, t, \tau)=\frac{1}{[4 \pi(t-\tau)]^{N / 2}} \cdot \exp \left\{\frac{-|x-y|^{2}}{4(t-\tau)}\right\}
$$

be the fundamental solution of the heat equation. Assume $\left(\tilde{u}_{0}, \tilde{v}_{0}\right)$ is a pair of initial data such that the solution of (1.1) blows up. Fix radially symmetric $v_{0}\left(\geq \tilde{v}_{0}\right)$ in $B_{R}$ and take $M_{0}>v_{0}(0)$. Let the minimum of $u_{0}\left(\geq \tilde{u}_{0}\right)$ be large such that $T$ is small and satisfies

$$
M_{0} \geq v_{0}(0)+\frac{\alpha-1}{\alpha-q-1}\left[(\alpha-1) \varepsilon e^{p v_{0}(0)}\right]^{-\frac{q}{\alpha-1}} e^{\beta M_{0}} \cdot T^{\frac{\alpha-q-1}{\alpha-1}}
$$

Consider the auxiliary problem

$$
\left\{\begin{array}{l}
\bar{v}_{t}=\Delta \bar{v}, \quad(x, t) \in B_{R} \times(0, T), \\
\frac{\partial \bar{v}}{\partial \eta}=\left[(\alpha-1) \varepsilon e^{p v_{0}(0)}\right]^{-\frac{q}{\alpha-1}} e^{\beta M_{0}}(T-t)^{-\frac{q}{\alpha-1}}, \quad(x, t) \in \partial B_{R} \times(0, T), \\
\bar{v}(x, 0)=v_{0}(x), \quad x \in B_{R} .
\end{array}\right.
$$

For $\alpha>q+1$ and by Green's identity [5], we have

$$
\begin{aligned}
\bar{v}(x, t)= & \int_{B_{R}} \Gamma(x, y, t, 0) \cdot v_{0}(y) d y+\int_{0}^{t} \int_{\partial B_{R}} \Gamma(x, y, t, \tau) \cdot \frac{\partial \bar{v}}{\partial \eta} \cdot d S_{y} \cdot d \tau \\
= & \int_{B_{R}} \Gamma(x, y, t, 0) \cdot v_{0}(y) d y \\
& +\int_{0}^{t} \int_{\partial B_{R}} \Gamma(x, y, t, \tau) \cdot\left[(\alpha-1) \varepsilon e^{p v_{0}(0)}\right]^{-\frac{q}{\alpha-1}} e^{\beta M_{0}}(T-\tau)^{-\frac{q}{\alpha-1}} \cdot d S_{y} \cdot d \tau \\
\leq & v_{0}(0)+\frac{\alpha-1}{\alpha-q-1}\left[(\alpha-1) \varepsilon e^{p v_{0}(0)}\right]^{-\frac{q}{\alpha-1}} e^{\beta M_{0}} T^{\frac{\alpha-q-1}{\alpha-1}} \\
\leq & M_{0},
\end{aligned}
$$

thus, $M_{0} \geq \bar{v}(x, t)$, for any $(x, t) \in B_{R} \times(0, T)$. So $\bar{v}$ satisfies

$$
\left\{\begin{array}{l}
\bar{v}_{t}=\Delta \bar{v}, \quad(x, t) \in B_{R} \times(0, T) \\
\frac{\partial \bar{v}}{\partial \eta} \geq\left[(\alpha-1) \varepsilon e^{p v_{0}(0)}\right]^{-\frac{q}{\alpha-1}} e^{\beta \bar{v}}(T-t)^{-\frac{q}{\alpha-1}}, \quad(x, t) \in \partial B_{R} \times(0, T), \\
\bar{v}(x, 0)=v_{0}(x), \quad x \in B_{R} .
\end{array}\right.
$$

Combining the radial symmetry and the monotonicity of the initial data with the estimate (3.2), we have

$$
u^{q}(|x|, t) \leq u^{q}(0, t) \leq\left[(\alpha-1) \varepsilon e^{p v_{0}(0)}\right]^{-\frac{q}{\alpha-1}}(T-t)^{-\frac{q}{\alpha-1}} \quad(x, t) \in B_{R} \times(0, T) .
$$

So, $v$ satisfies that

$$
\left\{\begin{array}{l}
v_{t}=\Delta v, \quad(x, t) \in B_{R} \times(0, T), \\
\frac{\partial v}{\partial \eta} \leq\left[(\alpha-1) \varepsilon e^{p v_{0}(0)}\right]^{-\frac{q}{\alpha-1}} e^{\beta v}(T-t)^{-\frac{q}{\alpha-1}}, \quad(x, t) \in \partial B_{R} \times(0, T), \\
v(x, 0)=v_{0}(x), \quad x \in B_{R} .
\end{array}\right.
$$


By the comparison principle, $v \leq \bar{v} \leq M_{0}$, so $v$ remains bounded up to time $T$. Since $\left(u_{0}, v_{0}\right) \geq\left(\tilde{u}_{0}, \tilde{v}_{0}\right),(u, v)$ blows up, hence only $u$ blows up at time $T$.

Secondly, we prove the necessity. Assume $u$ blows up while $v$ remains bounded, say $v \leq C$.

By Green's identity, we have

$$
u(0, t) \leq u(0, z)+C u^{\alpha}(0, t)(T-z)
$$

for any $z \in(0, T)$, take $t$ such that $u(0, t)=2 u(0, z)$, then

$$
u(0, z) \leq C u^{\alpha}(0, z)(T-z)
$$

hence,

$$
u(0, t) \geq C(T-t)^{-\frac{1}{\alpha-1}} \quad t \in(0, T) .
$$

For some $t_{1} \in(0, T)$, we can find a suitable $\varepsilon_{1} \in(0,1)$, such that

$$
u_{t}\left(x, t_{1}\right)-\varepsilon_{1}\left(u_{0}^{\alpha} e^{p v_{0}}\right)\left(x, t_{1}\right) \geq 0 .
$$

Similarly to Lemma 3.1, we can prove there must be

$$
\begin{aligned}
& u_{t}(x, t) \geq \varepsilon_{1}\left(u^{\alpha} e^{p v}\right)(x, t) \\
& v_{t}(x, t) \geq \varepsilon_{1}\left(u^{q} e^{\beta v}\right)(x, t)
\end{aligned} \quad(x, t) \in B_{R} \times\left[t_{1}, T\right) .
$$

Then

$$
v_{t}(0, t) \geq \varepsilon_{1} e^{\beta v_{0}(0)} C^{q}(T-t)^{-\frac{q}{\alpha-1}}, \quad t \in\left[t_{1}, T\right) .
$$

Integrating the above inequality from $t_{1}$ to $t$, we have

$$
\nu(0, t) \geq \varepsilon_{1} e^{\beta \nu_{0}(0)} C^{q} \int_{t_{1}}^{t}(T-\tau)^{-\frac{q}{\alpha-1}} d \tau+\nu\left(0, t_{1}\right) .
$$

The boundedness of $v$ requires that $\alpha>q+1$.

The following lemma proves the sufficient and necessary condition on the existence of $v$ blowing up alone.

Lemma 3.4 There exist suitable initial data such that $v$ blows up while u remains bounded if and only if $\beta>p$.

Proof Firstly, we prove the sufficiency. Assume $\left(\tilde{u}_{0}, \tilde{v}_{0}\right)$ is a pair of initial data such that the solution of (1.1) blows up. Fix radially symmetric $u_{0}\left(\geq \tilde{u}_{0}\right)$ in $B_{R}$ and take $M_{1}>u_{0}(0)$. Let the minimum of $v_{0}\left(\geq \tilde{v}_{0}\right)$ be large such that $T$ is small and satisfies

$$
M_{1} \geq u_{0}(0)+\frac{\beta}{\beta-p}\left[\beta \varepsilon u_{0}^{q}(0)\right]^{-\frac{p}{\beta}} M_{1}^{\alpha} T^{\frac{\beta-p}{\beta}} .
$$


Consider the auxiliary problem

$$
\left\{\begin{array}{l}
\bar{u}_{t}=\Delta \bar{u}, \quad(x, t) \in B_{R} \times(0, T), \\
\frac{\partial \bar{u}}{\partial \eta}=\left[\beta \varepsilon u_{0}^{q}(0)\right]^{-\frac{p}{\beta}} M_{1}^{\alpha}(T-t)^{-\frac{p}{\beta}}, \quad(x, t) \in \partial B_{R} \times(0, T), \\
\bar{u}(x, 0)=u_{0}(x), \quad x \in B_{R} .
\end{array}\right.
$$

For $\beta>p$, and by Green's identity, we have

$$
\bar{u}(x, t) \leq u_{0}(0)+\frac{\beta}{\beta-p}\left[\beta \varepsilon u_{0}^{q}(0)\right]^{-\frac{p}{\beta}} M_{1}^{\alpha} T^{\frac{\beta-p}{\beta}} \leq M_{1} .
$$

So $\bar{u}$ satisfies

$$
\frac{\partial \bar{u}}{\partial \eta} \geq\left[\beta \varepsilon u_{0}^{q}(0)\right]^{-\frac{p}{\beta}} \bar{u}^{\alpha}(T-t)^{-\frac{p}{\beta}}, \quad(x, t) \in \partial B_{R} \times(0, T) .
$$

From (3.3), we have

$$
\frac{\partial u}{\partial \eta} \leq\left[\beta \varepsilon u_{0}^{q}(0)\right]^{-\frac{p}{\beta}} u^{\alpha}(T-t)^{-\frac{p}{\beta}}, \quad(x, t) \in \partial B_{R} \times(0, T) .
$$

By the comparison principle, $u \leq \bar{u} \leq M_{1}$. Since $\left(u_{0}, v_{0}\right) \geq\left(\tilde{u}_{0}, \tilde{v}_{0}\right),(u, v)$ blows up, hence only $v$ blows up at time $T$.

Secondly, we prove the necessity. Assume $v$ blows up while $u$ remains bounded, say $u \leq C$.

By Green's identity, we have

$$
v(0, t) \leq v(0, z)+C e^{\beta \nu(0, t)}(T-z) .
$$

For any $z \in(0, T)$, take $t$ such that $v(0, t)=v(0, z)+1$, then

$$
C e^{\beta v(0, z)}(T-z) \geq 1
$$

thus

$$
v(0, t) \geq \ln [C(T-t)]^{-\frac{1}{\beta}}, \quad t \in(0, T) .
$$

From (3.5) and (3.6), we have

$$
u_{t}(0, t) \geq \varepsilon_{1} u_{0}^{\alpha}(0) C^{-\frac{p}{\beta}}(T-t)^{-\frac{p}{\beta}}, \quad t \in\left(t_{1}, T\right) .
$$

Integrating (3.7) from $t_{1}$ to $t$, we obtain that

$$
u(0, t) \geq u\left(0, t_{1}\right)+\varepsilon_{1} u_{0}^{\alpha}(0) C^{-\frac{p}{\beta}} \int_{t_{1}}^{t}(T-\tau)^{-\frac{p}{\beta}} d \tau
$$

The boundedness of $u$ requires that $\beta>p$.

\section{Coexistence of simultaneous and non-simultaneous blow-up}

In this section, we consider the coexistence of both simultaneous and non-simultaneous blow-ups. In order to prove Theorem 1.3, we introduce following lemma. 
Lemma 4.1 The set of $\left(u_{0}, v_{0}\right)$ in $\mathbb{V}_{0}$ such that $v$ blows up while $u$ remains bounded is open in $L^{\infty}$-topology.

Proof Let $(u, v)$ be a solution of (1.1) with initial data $\left(u_{0}, v_{0}\right) \in \mathbb{V}_{0}$ such that $v$ blows up at $T$ while $u$ remains bounded, that is $0<u(0, t) \leq M$. We only need to find a $L^{\infty}$-neighborhood of $\left(u_{0}, v_{0}\right)$ in $\mathbb{V}_{0}$, such that any solution $(\hat{u}, \hat{v})$ of $(1.1)$ coming from this neighborhood maintains the property that $\hat{v}$ blows up while $\hat{u}$ remains bounded.

By Lemma 3.4, we know $\beta>p$. Take $M_{2}>M+\frac{u_{0}(0)}{2}$, let $(\tilde{u}, \tilde{v})$ be the solution of the following problem:

$$
\left\{\begin{array}{l}
\tilde{u}_{t}=\Delta \tilde{u}, \quad \tilde{v}_{t}=\Delta \tilde{v}, \quad(x, t) \in B_{R} \times\left(0, T_{0}\right), \\
\frac{\partial \tilde{u}}{\partial \eta}=e^{p \tilde{\nu}} \tilde{u}^{\alpha}, \quad \frac{\partial \tilde{v}}{\partial \eta}=\tilde{u}^{q} e^{\beta \tilde{v}}, \quad(x, t) \in \partial B_{R} \times\left(0, T_{0}\right), \\
\tilde{u}(x, 0)=\tilde{u}_{0}(x), \quad \tilde{v}(x, 0)=\tilde{v}_{0}(x), \quad x \in B_{R},
\end{array}\right.
$$

where radially symmetric $\left(\tilde{u}_{0}, \tilde{v}_{0}\right)$ is to be determined and $T_{0}$ is the maximal existence time.

Denote

$$
\mathbb{N}\left(u_{0}, v_{0}\right)=\left\{\left(\tilde{u}_{0}, \tilde{v}_{0}\right) \in \mathbb{V}_{0} \mid\left\|\tilde{u}_{0}(0)-u\left(0, T-\varepsilon_{0}\right)\right\|_{\infty},\left\|\tilde{v}_{0}(0)-v\left(0, T-\varepsilon_{0}\right)\right\|_{\infty}<\frac{u_{0}(0)}{2}\right\} .
$$

Since $v$ blows up at time $T$, there exists small $\varepsilon_{0}>0$, such that $(\tilde{u}, \tilde{v})$ blows up and $T_{0}$ is small, satisfying

$$
M_{2}>M+\frac{u_{0}(0)}{2}+\frac{\beta}{\beta-p}\left[\beta \varepsilon\left(\frac{u_{0}(0)}{2}\right)^{q}\right]^{-\frac{p}{\beta}} T_{0}^{\frac{\beta-p}{\beta}} M_{2}^{\alpha}
$$

provided $\left(\tilde{u}_{0}, \tilde{v}_{0}\right) \in \mathbb{N}\left(u_{0}, v_{0}\right)$.

Consider the auxiliary system,

$$
\left\{\begin{array}{l}
\bar{u}_{t}=\Delta \bar{u}, \quad(x, t) \in B_{R} \times\left(0, T_{0}\right), \\
\frac{\partial \bar{u}}{\partial \eta}=\left[\beta \varepsilon \tilde{u}_{0}^{q}(0)\right]^{-\frac{p}{\beta}} M_{2}^{\alpha}\left(T_{0}-t\right)^{-\frac{p}{\beta}}, \quad(x, t) \in \partial B_{R} \times\left(0, T_{0}\right), \\
\bar{u}(x, 0)=\tilde{u}_{0}(x), \quad x \in B_{R} .
\end{array}\right.
$$

By Green's identity, $\bar{u} \leq M_{2}$. Hence,

$$
\frac{\partial \bar{u}}{\partial \eta} \geq\left[\beta \varepsilon \tilde{u}_{0}^{q}(0)\right]^{-\frac{p}{\beta}} \bar{u}^{\alpha}\left(T_{0}-t\right)^{-\frac{p}{\beta}}, \quad(x, t) \in \partial B_{R} \times\left(0, T_{0}\right) .
$$

Meanwhile, from (3.3), we get

$$
\tilde{v}(0, t) \leq \ln \left\{\left[\beta \varepsilon \tilde{u}_{0}^{q}(0)\right]^{-\frac{1}{\beta}}\left(T_{0}-t\right)^{-\frac{1}{\beta}}\right\} .
$$

So, we have

$$
\frac{\partial \tilde{u}}{\partial \eta} \leq\left[\beta \varepsilon \tilde{u}_{0}^{q}(0)\right]^{-\frac{p}{\beta}} \tilde{u}^{\alpha}\left(T_{0}-t\right)^{-\frac{p}{\beta}}, \quad(x, t) \in \partial B_{R} \times\left(0, T_{0}\right) .
$$

By the comparison principle, $\tilde{u} \leq \bar{u} \leq M_{2}$, then $\tilde{v}$ must blow up. 
According to the continuity with respect to initial data for bounded solutions, there must exist a neighborhood of $\left(u_{0}, v_{0}\right)$ in $\mathbb{V}_{0}$ such that every solution $(\hat{u}, \hat{v})$ starting from the neighborhood, will enter $\mathbb{N}\left(u_{0}, v_{0}\right)$ at time $T-\varepsilon_{0}$, and keeps the property that $\hat{v}$ blows up while $\hat{u}$ remains bounded.

Similarly, we can prove the set of $\left(u_{0}, v_{0}\right)$ in $\mathbb{V}_{0}$ such that $u$ blows up while $v$ remains bounded is open in $L^{\infty}$-topology, we omit the proof here.

Now, we give the proof of Theorem 1.3.

Proof of Theorem 1.3 Under our assumptions, from Lemma 3.3, we know that the set of $\left(u_{0}, v_{0}\right)$ in $\mathbb{V}_{0}$ such that $u$ blows up and $v$ remains bounded is nonempty. And from Lemma 3.4, we also know the set of $\left(u_{0}, v_{0}\right)$ in $\mathbb{V}_{0}$ such that $v$ blows up and $u$ is bounded is nonempty.

Moreover, Lemma 4.1 shows that such sets are open. Clearly, the two open sets are disjoint. That is to say, there exists $\left(u_{0}, v_{0}\right)$ such that $u$ and $v$ blow up simultaneously at a finite time $T$.

\section{Competing interests}

The authors declare that they have no competing interests.

\section{Authors' contributions}

The authors declare that the work was realized in collaboration with the same responsibility. All authors read and approved the final manuscript.

\section{Author details}

${ }^{1}$ Department of Mathematics, Jiangxi Vocational College of Finance and Economics, Jiujiang, Jiangxi 332000, P.R. China.

${ }^{2}$ Department of Information Engineering, Jiangxi Vocational College of Finance and Economics, Jiujiang, Jiangxi 332000, P.R. China.

\section{Acknowledgements}

We would like to thank the referees for their valuable comments and suggestions.

Received: 16 April 2012 Accepted: 25 July 2012 Published: 6 August 2012

References

1. Bebernes, J, Eberly, D: Mathematical Problem from Combustion Theory. Appl. Math. Sci., vol. 83. Springer, Berlin (1989)

2. Brändle, C, Quirós, F, Rossi, JD: Non-simultaneous blow-up for a quasilinear parabolic system with reaction at the boundary. Commun. Pure Appl. Anal. 4(3), 523-536 (2005)

3. Brändle, C, Quirós, F, Rossi, JD: The role of non-linear diffusion in non-simultaneous blow-up. J. Math. Anal. Appl. 308, 92-104 (2005)

4. Deng, K: Blow-up rates for parabolic systems. Z. Angew. Math. Phys. 47, 132-143 (1996)

5. Friedman, A: Partial Differential Equations of Parabolic Type. Prentice Hall, Englewood Cliffs (1964)

6. Lieberman, GM: Second Order Parabolic Differential Equations. World Scientific, Singapore (1996)

7. Lin, ZG, Xie, CH: The blow-up rate for a system of heat equations with nonlinear boundary conditions. Nonlinear Anal. 34, 767-778 (1998)

8. Liu, BC, Li, FJ: Blow-up properties for heat equations coupled via different nonlinearities. J. Math. Anal. Appl. 347, 294-303 (2008)

9. Liu, BC, Li, FJ: Non-simultaneous blow-up and blow-up rates for reaction-diffusion equations. Nonlinear Anal. 13, 764-778 (2012)

10. Pedersen, M, Lin, ZG: Blow-up estimates of the positive solution of a parabolic system. J. Math. Anal. Appl. 255 , 551-563 (2001)

11. Pinasco, JP, Rossi, JD: Simultaneous versus non-simultaneous blow-up. N.Z. J. Math. 29, 55-59 (2000)

12. Quirós, F, Rossi, JD: Non-simultaneous blow-up in a semilinear parabolic system. Z. Angew. Math. Phys. 52(2), $342-346$ (2001)

13. Quirós, F, Rossi, JD: Non-simultaneous blow-up in a nonlinear parabolic system. Adv. Nonlinear Stud. 3(3), 397-418 (2003)

14. Rossi, JD: The blow-up rate for a system of heat equations with non-trivial coupling at the boundary. Math. Methods Appl. Sci. 20, 1-11 (1997)

15. Rossi, JD, Souplet, P: Coexistence of simultaneous and non-simultaneous blow-up in a semilinear parabolic system. Differ. Integral Equ. 18, 405-418 (2005) 
16. Song, XF: Blow-up analysis for a system of heat equations with nonlinear flux which obey different laws. Nonlinear Anal. 69, 1971-1980 (2008)

17. Wang, MX: Parabolic systems with a nonlinear boundary conditions. Chin. Sci. Bull. 40(17), 1412-1414 (1995)

18. Zhao, LZ, Zheng, SN: Blow-up estimates for system of heat equations coupled via nonlinear boundary flux. Nonlinear Anal. 54, 251-259 (2003)

19. Zheng, SN, Qiao, L: Non-simultaneous blow-up in a reaction-diffusion system. Appl. Math. Comput. 180, 309-317 (2006)

20. Zheng, SN, Liu, BC, Li, FJ: Non-simultaneous blow-up for a multi-coupled reaction-diffusion system. Nonlinear Anal. 64, 1189-1202 (2006)

doi:10.1186/1687-2770-2012-85

Cite this article as: Xu and Zeng: Non-simultaneous blow-up for a parabolic system with nonlinear boundary flux which obey different laws. Boundary Value Problems 2012 2012:85.

Submit your manuscript to a SpringerOpen ${ }^{\circ}$ journal and benefit from:

- Convenient online submission

- Rigorous peer review

- Immediate publication on acceptance

- Open access: articles freely available online

- High visibility within the field

- Retaining the copyright to your article

Submit your next manuscript at $>$ springeropen.com 